\title{
MODELLING OF EQUIVALENT MASS AND RIGIDITY OF CONTINUAL SEGMENT OF THE INTER-RESONANCE VIBRATION MACHINE
}

\author{
O.S. LANETS, V.T. DMYTRIV*and O.Yu. KACHUR \\ Department of Mechanics and Automation of Mechanical Engineering \\ Institute of Engineering Mechanics and Transport, Lviv Polytechnic National University, \\ 1 Profesorska St., Lviv, 79013, UKRAINE \\ E-mail: Dmytriv_V@lukr.net \\ I.A. DEREVENKO \\ Department of Strength of Materials and Structural Mechanics, \\ Institute of Civil Engineering and Building Systems, Lviv Polytechnic National University \\ 6 Karpinskoho St., Lviv, 79013, UKRAINE \\ Yu.Ya. NOVITSKYI \\ Department of Mechanical Engineering Technologies \\ Institute of Engineering Mechanics and Transport, Lviv Polytechnic National University \\ 1 Profesorska St., Lviv, 79013, UKRAINE \\ I.M. HORODETSKYY \\ Department of Project Management and Occupational Safety \\ Faculty of Mechanic and Power Engineering, Lviv National Agrarian University \\ Lviv-Dubliany, 80381, UKRAINE
}

\begin{abstract}
The article deals with a continual segment of an inter-resonance vibration machine. In the form of a solid with distributed parameters this segment combines two defining parameters, namely: the inertial parameter of reactive: masses and appropriate rigidity of elastic coupling. These operation factors are revealed only in dynamic processes and are clearly not included in the parameters of the continual segment. Analytical dependences are developed for modeling of defining parameters of an inter-resonance system, namely: reactive mass and appropriate rigidity of elastic: coupling. Parameters of the reference point of the continual segment passing through its center of velocity are studied. The inertial parameter of the reactive mass and the rigidity of elastic coupling were modeled by the Rayleigh-Ritz method. The reliability of the results of theoretical research was confirmed experimentally and the parameters of the partial frequency of the continual segment were determined.
\end{abstract}

Key words: three-mass vibration system, Rayleigh-Ritz method, continual segment, equivalent mass, equivalent rigidity.

\section{Introduction}

One of the promising directions of development of vibrating technological equipment is the creation of inter-resonant vibration machines. Inter-resonant modes of operation provide a significant reduction in power consumption in the drives. Lanets et al. [1] supposes that for the efficient use of energy-saving interresonant modes of operation it is necessary to use small inertial values of reactive mass and rigidity of the corresponding elastic assembly. Such properties are characteristic of a flexible body - a continuous system that

\footnotetext{
${ }^{*}$ To whom correspondence should be addressed
} 
optimally combines inertial and rigid parameters.

It is known that a straight rod or beam has many natural forms of vibration, where each form of vibration corresponds to a certain value of frequency. Gursky et al. [2] studied the natural frequency of beam vibrations by the finite element method. However, this method can create a number of errors, which forces researchers to vary the complexity (density) of the structural grid to obtain acceptable results. In the work of Sharma et al. [3] the vibration of functionally graduated plates was analyzed using the finite element method. Jaworski et al. [4] conducted the experiment using the finite element method in ANSYS software. Raju et al. [5] analyzed the large amplitudes of free vibrations of conical beams using the continuum and the finite element method. Gharaibeh et al. [6] investigated the natural vibrations of rectangular plates with partially clamped edges (at the corners) by the finite element method.

Srinivasa et al. [7] experimentally studied the free vibration frequencies of isotropic and laminated composite plates. Jaroszewicz [8] analyzed the vibration of homogeneous and isotropic circular thin plates with nonlinear variable thickness, which are clamped at the edges. Zur [9] considered the natural vibration of homogeneous and isotropic circular thin plates with variable distributed parameters using Green's functions, which depend on the Poisson coefficient and the coefficient of distribution of the rigidity of the plate on the bend. In the works of Amabili et al. [10], analytical dependences were obtained using the nonlinear Von Karman plate theory and global sampling, as well as large amplitudes of forced vibrations of a thin rectangular plate with different concentrated masses were experimentally studied.

In Buchacz articles [11, 12], the beam is considered as a homogeneous beam with constant length parameters and the graph theory is used to establish the natural frequency of vibrations. Clementi et al. [13] studied the frequency response curves of a non-uniform beam by the MTS asymptotic expansion method. This non-uniform beam oscillates nonlinearly. Using the multiple time scale method, in which axial inertia is neglected, the equations of motion are statically compressed only at the transverse displacement. Firouz-Abadi et al. [14] presented an analytical solution based on the approximation of Wentzel, Kramers, Brillouin for free transverse vibrations of beams of various cross sections. Ece et al. [15] solved the problem of vibration of beams with exponentially variable cross-sectional width for three different types of boundary conditions: free, jointed and clamped ends. Mahmoud et al. [16] analyzed nonlinear free vibrations (of large amplitudes) of conical rods using the Max-Min Approach and Homotopy Perturbation Method. After comparing the obtained results, the authors confirmed the convergence of vibration. Kisa et al. [17] proposed a method for determining the frequency of natural vibrations of a uniform and stepped cracked beam with a circular cross section. Free vibrations of tapered beams were considered by Lee et al. [18]. The authors solved differential equations by numerical methods and established natural frequencies by combining the Runge Kutta method and the determinant search method. Shin et al. [19] applied the generalized method of differential quadrature and the method of differential transformation to the vibrational analysis of circular arches, declaring rapid convergence and accuracy. Lee [20] developed a method for finding the natural vibration of a Bernoulli-Euler tapered beam, in which the roots of the differential equation were determined using the Frobenius method, which allows obtaining solutions of the power series for bending vibrations.

The analysis confirms the relevance of further research in the direction of studying the natural frequencies of continual systems.

Analytical study of natural frequency by the Ritz method was considered in Vescovini et al. [21], where free vibrations and stability losses in highly anisotropic plates were studied. Dozio [22] expanded the potential of the Ritz method for predicting the natural bending frequencies of plates with various complicating factors. Study results confirmed the efficiency and accuracy of the method.

The Rayleigh-Ritz method ensures sufficient accuracy of the results (Yuan et al. [23], Kumar [24], Rahbar-Ranji et al. [25], Mazanoglu [26], Babakov [27]). This method can be applied to a wide range of plates, bars, rods, beams, etc., with any aspect ratio. Due to its versatility and accuracy, the Rayleigh-Ritz method is used to study the natural vibration frequency in this article.

\section{Description of the problem}

According to known methods for calculating inter-resonance vibration machines, Lanets et al. set five defining parameters of the vibration system (Fig.1a), namely: the inertial values of the three masses of $m_{1}, m_{2}$ 
and $m_{3}$ and the values of $c_{12}$ and $c_{23}$ rigidity of the elastic units, connecting the three vibrating masses, respectively. The oscillating system is driven with $\omega$ circular frequency of forced vibrations by a crank mechanism with the $\varepsilon$ eccentricity, where the $F_{0}$ perturbation force is $\varepsilon c_{23}$. The vibration system bases on the foundation through vibration insulators with $c_{i s}$ rigidity.

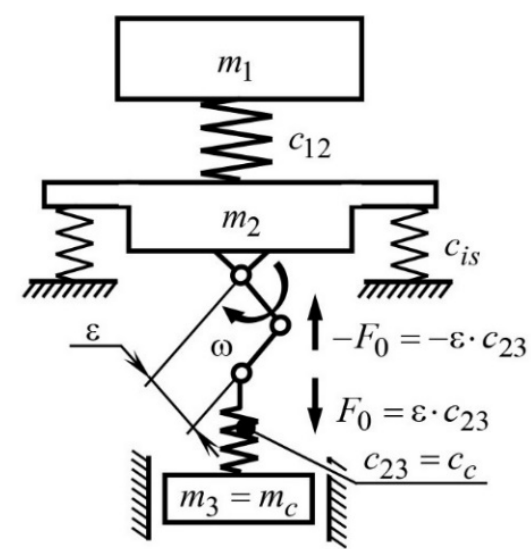

(a)

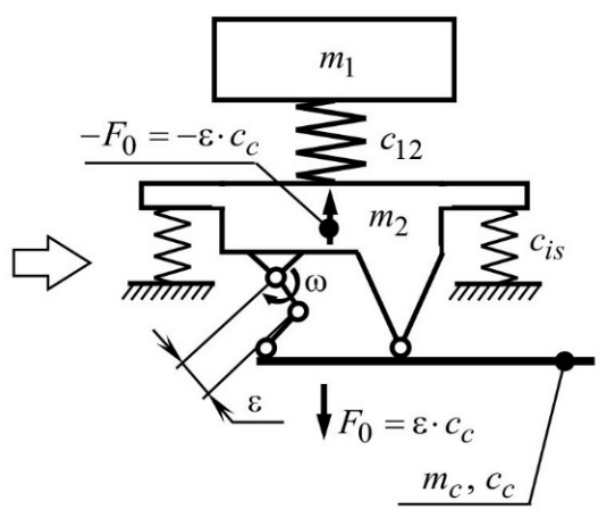

(b)

Fig.1. Schematic structures of three-mass mechanical vibration systems with eccentric drive: a - discrete; $\mathrm{b}$ - discrete-continual

The problem is that in the discrete-continual system (Fig.1b) the continual segment (beam) simultaneously connects two parameters - mass of $m_{3}$ and rigidity of $c_{23}$, which are revealed only in dynamic processes and are clearly not included in the parameters of the beam.

The task of this article is to develop analytical dependences that will make it possible to study the influence of two defining parameters of the inter-resonance system, namely: the mass of $m_{3}$ and rigidity of $c_{23}$, which are summary values of the inertial parameter of $m_{c}$ and rigidity of $c_{c}$ of the beam taking into consideration that $m_{3} \equiv m_{c}$, and $c_{23} \equiv c_{c}$.

\section{Results and discussions}

\subsection{The reference point of the continual segment}

To determine the consolidated rigidity of $c_{c}$ and the consolidated mass $m_{c}$ of the beam in vibrating motion, the point of reference at the certain coordinate of $X_{c}$ is analytically determined. This point is formed as the interaction of the $X_{c 1}, X_{c 2}$ and $X_{c 3}$ points of reference respectively, the left, middle and right segments of the beam, the lengths of which are $L_{1}, L_{2}$ and $L_{3}$ ( $L$ is the length of the beam). It is at this point that the inertial value and rigidity of the entire beam are concentrated by convention (Fig.2).

There may be many reference points of the beam. For each case, we can find a specific value of mass and rigidity and enter them into a discrete model.

A question arises as to how adequate these values are and whether they are really perceived by the vibration system.

We assume that the discretized inter-resonance vibration system perceives the continual segment with distributed parameters discretely just concerning the consolidated or reference point at the $X_{c}$ certain coordinate relative to the center of velocities of the beam (Fig.2). Mathematically, the velocity center is defined 
as the ratio of the sum of the static moments of the velocities of each segment to the sum of the velocities along the entire length of the beam. For amplitude values, this can be written in integral form as follows:

$$
X_{c}=\frac{\omega X_{c 1} \int_{0}^{L_{l}} w_{l}(x) \mathrm{d} x+\omega X_{c 2} \int_{L_{1}}^{L_{1}+L_{2}} w_{m}(x) \mathrm{d} x+\omega X_{c 3} \int_{L_{l}+L_{2}}^{L} w_{r}(x) \mathrm{d} x}{\omega \int_{0}^{L_{l}} w_{l}(x) \mathrm{d} x+\omega \int_{L_{1}}^{L_{1}+L_{2}} w_{m}(x) \mathrm{d} x+\omega \int_{L_{1}+L_{2}}^{L} w_{r}(x) \mathrm{d} x} .
$$

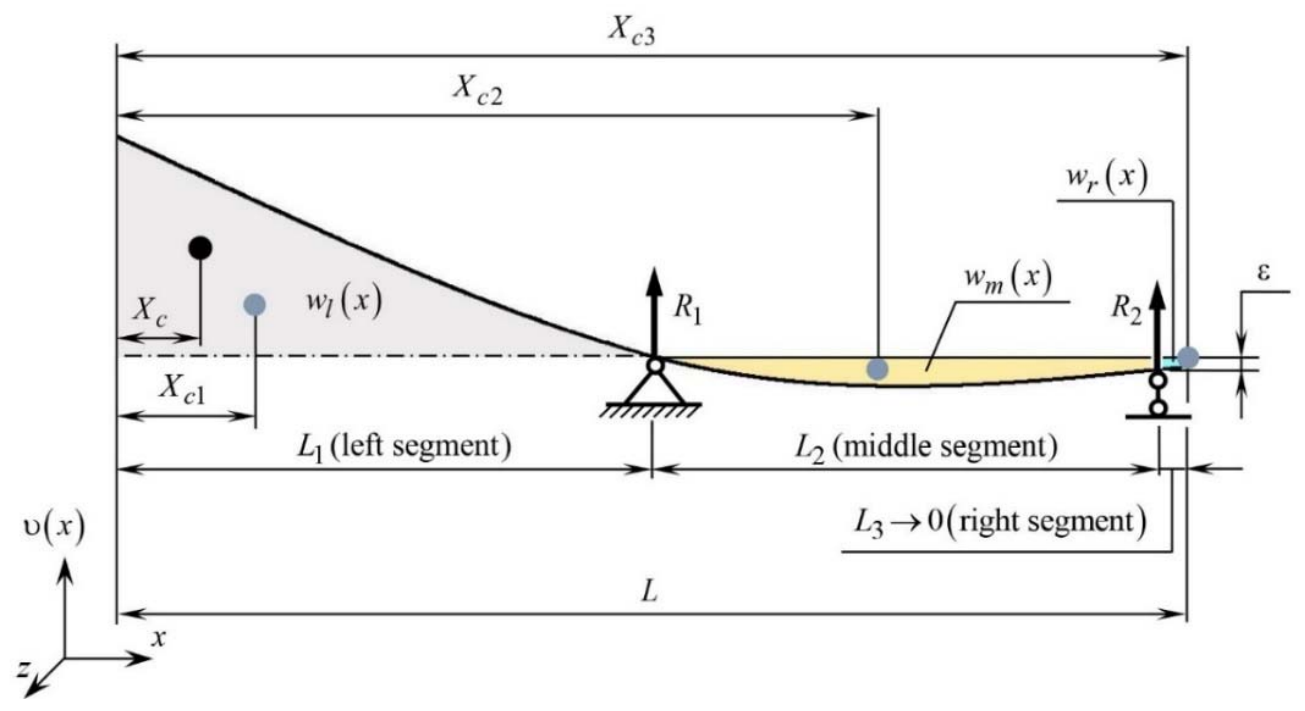

Fig.2. The scheme of velocities of the $v(x)=\omega w(x)$ rod segments deflection in the $x$ coordinate, where: $R_{l}$, $R_{2}$ - reaction of supports; $w_{l}(x), w_{m}(x)$ and $w_{r}(x)$-deflection of the beam in the left, middle and right segments respectively.

Velocity centers of $X_{c 1}, X_{c 2}$ and $X_{c 3}$ individual sections are searched according to:

$$
X_{c l}=\frac{\omega \int_{0}^{L_{l}} x \cdot w_{l}(x) \mathrm{d} x}{\omega \int_{0}^{L_{l}} w_{l}(x) \mathrm{d} x} ; \quad X_{c 2}=\frac{\omega \int_{L_{1}}^{L_{1}+L_{2}} x \cdot w_{m}(x) \mathrm{d} x}{\omega \int_{L_{1}}^{L_{1}+L_{2}} w_{m}(x) \mathrm{d} x} ; \quad X_{c 3}=\frac{\omega \int_{L_{1}+L_{2}}^{L} x \cdot w_{r}(x) \mathrm{d} x}{\omega \int_{L_{1}+L_{2}}^{L} w_{r}(x) \mathrm{d} x} .
$$

After substituting Eqs (3.2) into Eq.(3.1) and simplifying the obtained expression, the equation for determining the center of velocity of the elastic beam, which is analogous to finding the center of mass of the solid, will take the form of: 


$$
X_{c}=\frac{\int_{0}^{L_{l}} x \cdot w_{l}(x) \mathrm{d} x+\int_{L_{1}}^{L_{1}+L_{2}} x \cdot w_{m}(x) \mathrm{d} x+\int_{L_{1}+L_{2}}^{L} x \cdot w_{r}(x) \mathrm{d} x}{\int_{0}^{L_{l}} w_{l}(x) \mathrm{d} x+\int_{L_{l}}^{L_{1}+L_{2}} w_{m}(x) \mathrm{d} x+\int_{L_{1}+L_{2}}^{L} w_{r}(x) \mathrm{d} x} .
$$

Knowing the $X_{c}$ coordinate of the reference point, the equivalent mass and equivalent rigidity of the elastic beam can be determined.

\subsection{Modeling of equivalent mass and equivalent rigidity by Rayleigh-Ritz method}

The method is based on the fact that the amplitude values of the $\mathrm{K}_{r}$ kinetic and potential $\Pi_{r}$ energies of the elastic beam for the period of vibrations are the same. That is:

$$
\mathrm{K}_{r}=\Pi_{r} .
$$

The $\mathrm{K}_{r}$ kinetic energy of the beam consists of the kinetic energy from the $w(x)$ displacement and from the rotation at the $\theta(x)$ angle:

$$
\mathrm{K}_{r}=\frac{1}{2} \int_{0}^{L} m_{r r} \omega^{2} w(x)^{2} \mathrm{~d} x+\frac{1}{2} \int_{0}^{L} J_{r r}(x) \omega^{2}\left(\frac{\mathrm{d} w(x)}{\mathrm{d} x}\right)^{2} \mathrm{~d} x,
$$

where $m_{r r}$ is the linear mass of beam; $w(x)$ is the displacement of the cross section of the rod in the vertical direction depending on the $x$ coordinate (along the length of the elastic beam); $J_{r r}$ is the moment of inertia of beam length unit (linear unit) as the function of $x$ coordinate.

Considering the solid as a beam, the moment of inertia of the unit length of the beam is written as:

$$
J_{r r}(x)=\frac{\rho h b_{r}}{3} x^{2}=\frac{m_{r r}}{3} x^{2}
$$

where $\rho$ is the unit weight for beam material; $h$ is the width of the elastic beam; $b_{r}$ is the thickness of the elastic beam.

The displacement of the cross section of the rod of $w(x)$ in general can be represented as [27]:

$$
w(x)=A \cos (\xi x)+B \sin (\xi x)+C \operatorname{ch}(\xi x)+D \operatorname{sh}(\xi x)
$$

where $A, B, C$ and $D$ - four arbitrary constants are chosen so that for Eq.(3.7) the boundary conditions are satisfied, i.e. the conditions of fixing the ends of the beam (segments of the beam);

$$
\xi=\sqrt[4]{\frac{m_{r r} \omega^{2}}{E J_{z}}}
$$

where $E$ is the (tensile) elastic modulus; $J_{z}$ is moment of inertia of beam rectangular cross-section area 
relative to the neutral line of the section; $E J_{z}$ is the rigidity of the beam cross-section area.

The $\Pi_{r}$ potential energy of the bent axis of the rod was determined as the sum of the potential energy of its bending and the potential energy of shear in the layers of the rod and the result can be written as:

$$
\Pi_{r}=\frac{1}{2} E J_{z} \int_{0}^{L}\left(\frac{\mathrm{d}^{2} w(x)}{\mathrm{d} x^{2}}\right)^{2} \mathrm{~d} x+\frac{1}{2} \frac{k_{y}\left(E J_{z}\right)^{2}}{G F_{r}} \int_{0}^{L}\left(\frac{\mathrm{d}^{3} w(x)}{\mathrm{d} x^{3}}\right)^{2} \mathrm{~d} x
$$

where $G$ is the modulus of rigidity; $F_{r}$ is the cross-section area of the beam (constant along the length of the beam); $k_{y}$ is the coefficient that depends on the shape of the cross section of the beam [27].

According to formula Eq.(3.4), after a comparison Eq.(3.5) and Eq.(3.9) and selection from each term of the equation of the amplitude of vibration at the $w\left(X_{c}\right)$ reference point which is a constant value, the result can be written as:

$$
\begin{aligned}
& \frac{\omega^{2} w\left(X_{c}\right)^{2}}{2}\left[\frac{m_{r r}}{w\left(X_{c}\right)^{2}} \int_{0}^{L} w(x)^{2} \mathrm{~d} x+\frac{1}{w\left(X_{c}\right)^{2}} \int_{0}^{L} J_{r r}(x)\left(\frac{\mathrm{d} w(x)}{\mathrm{d} x}\right)^{2} \mathrm{~d} x\right]= \\
= & \frac{w\left(X_{c}\right)^{2}}{2}\left[\frac{E J_{z}}{w\left(X_{c}\right)^{2}} \int_{0}^{L}\left(\frac{\mathrm{d}^{2} w(x)}{\mathrm{d} x^{2}}\right)^{2} \mathrm{~d} x+\frac{k_{y}\left(E J_{z}\right)^{2}}{w\left(X_{c}\right)^{2} G F_{r}} \int_{0}^{L}\left(\frac{\mathrm{d}^{3} w(x)}{\mathrm{d} x^{3}}\right)^{2} \mathrm{~d} x\right] .
\end{aligned}
$$

Equation (3.10) is the equality of kinetic and potential energy. Given that the $\omega \cdot w\left(X_{c}\right)$ is a linear velocity of $v\left(X_{c}\right)$, Eq.(3.10) will take the form:

$$
\frac{v\left(X_{c}\right)^{2} \cdot m_{c}}{2}=\frac{w\left(X_{c}\right)^{2} \cdot c_{c}}{2}
$$

where

$$
\begin{aligned}
& m_{c}=\frac{m_{r r}}{w\left(X_{c}\right)^{2}} \int_{0}^{L} w(x)^{2} \mathrm{~d} x+\frac{1}{w\left(X_{c}\right)^{2}} \int_{0}^{L} J_{r r}(x)\left(\frac{\mathrm{d} w(x)}{\mathrm{d} x}\right)^{2} \mathrm{~d} x, \\
& c_{c}=\frac{E J_{z}}{w\left(X_{c}\right)^{2}} \int_{0}^{L}\left(\frac{\mathrm{d}^{2} w(x)}{\mathrm{d} x^{2}}\right)^{2} \mathrm{~d} x+\frac{k_{y}\left(E J_{z}\right)^{2}}{w\left(X_{c}\right)^{2} G F_{r}} \int_{0}^{L}\left(\frac{\mathrm{d}^{3} w(x)}{\mathrm{d} x^{3}}\right)^{2} \mathrm{~d} x
\end{aligned}
$$

are equivalent mass and equivalent rigidity, respectively, of the elastic beam with distributed parameters at the $X_{c}$ point of reduction.

\subsection{Model of the continual segment}

The continual segment is considered as the beam with several sections. Three independent sections will be connected by the $R_{1}$ and $R_{2}$ reactions in the supports. The left end of the rod, which is in the free state, is taken as the starting point (Fig.3).

Since the left end of the beam is free, the $w(x)$ cross motion and the $\theta(x)=w^{\prime}(x)$ angle of rotation 
are in it, and the $M(x)=E J_{z} w^{\prime \prime}(x)$ bending moment and $Q(x)=E J_{z} w^{\prime \prime \prime}(x)$ transverse force are absent. The equations of $w_{l}(x), w_{m}(x)$ in $w_{r}(x)$ deflections in the left, middle and right beam sections, respectively, will take the form:

$$
\begin{aligned}
& w_{l}(x)=\frac{A}{2}(\operatorname{ch}(\xi x)+\cos (\xi x))+\frac{B}{2}(\operatorname{sh}(\xi x)+\sin (\xi x)), \\
& w_{m}(x)=w_{l}(x)+\frac{R_{1}}{2 \xi^{3} E J_{z}} \cdot\left(\operatorname{sh}\left(\xi\left(x-L_{l}\right)\right)-\sin \left(\xi\left(x-L_{1}\right)\right)\right), \\
& w_{r}(x)=w_{l}(x)+w_{m}(x)+\frac{R_{2}}{2 \xi^{3} E J_{z}} \cdot\left(\operatorname{sh}\left(\xi\left(x-\left(L-L_{2}\right)\right)\right)-\sin \left(\xi\left(x-\left(L-L_{2}\right)\right)\right)\right) .
\end{aligned}
$$

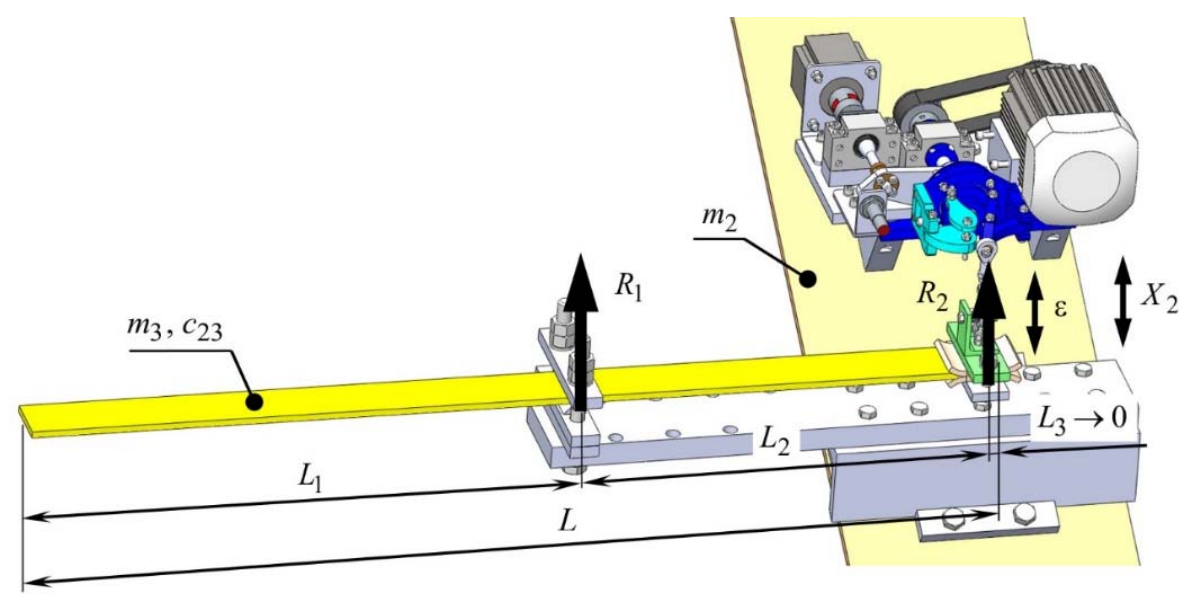

Fig.3. Calculation scheme of the beam continual segment.

Given that for the left section in the $R_{1}$ support the displacement is $\delta_{1}=X_{2}\left(X_{2}-\right.$ is the amplitude of vibration of the $m_{2}$ intermediate mass) at $x=L_{1}$ (Fig.3), and for the middle section ( $R_{2}$ support) the displacement is $\delta_{2}=X_{2}+\varepsilon$ at $x=L_{1}+L_{2}$, the system of four equations of forced vibration of the beam will be:

$$
\left\{\begin{array}{l}
w_{r}{ }^{\prime \prime}(L)=0, \\
w_{r}{ }^{\prime \prime}(L)=0, \\
w_{l}\left(L_{1}\right)=\delta_{1}, \\
w_{m}\left(L_{1}+L_{2}\right)=\delta_{2} .
\end{array}\right.
$$

\subsection{Results of modeling of equivalent mass and equivalent rigidity}

It is established that to ensure $\zeta=5 g$ overload ( $g$ - acceleration of gravity) at the frequency of $\omega_{f}=99.484 \mathrm{rad} / \mathrm{s}\left(n_{f}=950 \mathrm{rpm}\right)$ forced vibrations the amplitude of vibration of the working body should 
be $X_{1}=4.955 \cdot 10^{-3} \mathrm{~m}$, and the intermediate mass of $X_{2}=-5.868 \cdot 10^{-3} \mathrm{~m}$. As above determined $X_{1}$ and $X_{2}$ parameters were substituted into Eqs (3.17), the values of $A$ and $B$ constants, the reactions of the $R_{1}$ and $R_{2}$ supports were determined, which are: $A=0.083, B=-0.066, R_{l}=-592.066 \mathrm{~N}$ and $R_{l}=341.729 \mathrm{~N}$. After determined values were substituted in Eq.(3.3), the coordinate of the reference point along the $x$-axis was defined which is $X_{c}=0.01 \mathrm{~m}$.

To establish the equivalent mass and the equivalent rigidity of the beam according to expressions (3.12) and (3.13), these parameters will be formed as an algebraic sum of the corresponding indices on each section of the beam, i.e.:

$$
\begin{aligned}
& c_{c}=c_{c 1}+c_{c 2}+c_{c 3}+c_{c 1 i}+c_{c 2 i}+c_{c 3 i}, \\
& m_{c}=m_{c 1}-m_{c 2}-m_{c 3}+m_{c 1 i}-m_{c 2 i}-m_{c 3 i}
\end{aligned}
$$

where $c_{c 1}, c_{c 2}, c_{c 3}$ - fractions of equivalent rigidities of the beam bending on left, middle and right sections, respectively; $c_{c 1 i}, c_{c 2 i}, c_{c 3 i}$ - fractions of equivalent rigidities of the beam bending on left, middle and right sections, respectively; $m_{c 1}, m_{c 2}, m_{c 3}$ - fractions of equivalent mass of linear movement of the beam on left, middle and right segments, respectively; $m_{c l i}, m_{c 2 i}, m_{c 3 i}$ - fractions of equivalent mass of the beam twist on left, middle and right sections, respectively. The values of the fractions of the equivalent masses and rigidities in the third section can be neglected due to the scantiness of its length.

To establish the fraction of the $m_{c l}$ equivalent mass of movement at the left end of the beam (from 0 to $L_{1}$ ), the left part of expression (3.12) and expression (3.14) were used:

$$
m_{c l}=m_{r r} \int_{0}^{L_{l}}\left(\frac{w_{l}(x)}{w\left(X_{c}\right)}\right)^{2} \mathrm{~d} x=0.243 \mathrm{~kg}
$$

where

$$
w\left(X_{c}\right)=\frac{A}{2}\left(\operatorname{ch}\left(\xi X_{c}\right)+\cos \left(\xi X_{c}\right)\right)+\frac{B}{2}\left(\operatorname{sh}\left(\xi X_{c}\right)+\sin \left(\xi X_{c}\right)\right)
$$

is the deflection of the beam at the point of reference.

On the middle section of the beam (from $L_{1}$ to $L_{1}+L_{2}$ ) we can determine the fraction of the equivalent mass of movement using Eq.(3.15):

$$
m_{c 2}=m_{r r} \int_{L_{1}}^{L_{1}+L_{2}}\left(\frac{w_{m}(x)}{w\left(X_{c}\right)}\right)^{2} \mathrm{~d} x=9.27 \cdot 10^{-3} \mathrm{~kg} .
$$

To establish the fraction of the $m_{c l i}$ equivalent mass of twist at the left end of the beam (from 0 to $L_{1}$ ) the right part of expression (3.12) and expression (3.14) were used:

$$
m_{c l i}=\int_{0}^{L_{l}}\left(\frac{m_{r r}}{3} x^{2}\right)\left(\frac{\mathrm{d}}{\mathrm{d} x}\left(\frac{w_{l}(x)}{w\left(X_{c}\right)}\right)\right)^{2} \mathrm{~d} x=0.082 \mathrm{~kg} .
$$


On the middle section of the beam (from $L_{1}$ to $L_{1}+L_{2}$ ) we can determine the fraction of the equivalent mass of twist using Eq.(3.15):

$$
m_{c 2 i}=\int_{L_{1}}^{L_{1}+L_{2}}\left(\frac{m_{r r}}{3}\left(x-L_{l}\right)^{2}\right)\left(\frac{\mathrm{d}}{\mathrm{d} x}\left(\frac{w_{m}(x)}{w\left(X_{c}\right)}\right)\right)^{2} \mathrm{~d} x=2.22 \cdot 10^{-3} \mathrm{~kg} .
$$

To establish the fraction of the $c_{c 1}$ equivalent rigidity of bending at the left end of the beam (from 0 to $L_{1}$ ), the left part of expression (3.13) and expression (3.14) were used:

$$
c_{c l}=E J_{z} \int_{0}^{L_{l}}\left(\frac{\mathrm{d}^{2}}{\mathrm{~d} x^{2}}\left(\frac{w_{l}(x)}{w\left(X_{c}\right)}\right)\right)^{2} \mathrm{~d} x=1.33 \cdot 10^{3} \mathrm{~N} / \mathrm{m} .
$$

On the middle section of the beam (from $L_{1}$ to $L_{1}+L_{2}$ ) we can determine the fraction of the equivalent rigidity of bending using Eq.(3.15):

$$
c_{c 2}=E J_{z} \int_{L_{1}}^{L_{1}+L_{2}}\left(\frac{\mathrm{d}^{2}}{\mathrm{~d} x^{2}}\left(\frac{w_{m}(x)}{w\left(X_{c}\right)}\right)\right)^{2} \mathrm{~d} x=1.416 \cdot 10^{3} \mathrm{~N} / \mathrm{m} .
$$

To establish the fraction of the $c_{c l i}$ equivalent rigidity of beam layers shearing at the left end of the beam (from 0 to $L_{1}$ ), the right part of expression (3.13) and expression (3.14) were used:

$$
c_{c l i}=\frac{k_{y}\left(E J_{z}\right)^{2}}{G F_{r}} \int_{0}^{L_{1}}\left(\frac{\mathrm{d}^{3}}{\mathrm{~d} x^{3}}\left(\frac{w_{l}(x)}{w\left(X_{c}\right)}\right)\right)^{2} \mathrm{~d} x=0.237 \mathrm{~N} / \mathrm{m} .
$$

On the middle section of the beam (from $L_{1}$ to $L_{1}+L_{2}$ ) we can determine the fraction of the equivalent rigidity of beam layers shearing using Eq.(3.15):

$$
c_{c 2 i}=\frac{k_{y}\left(E J_{z}\right)^{2}}{G F_{r}} \int_{L_{1}}^{L_{1}+L_{2}}\left(\frac{\mathrm{d}^{3}}{\mathrm{~d} x^{3}}\left(\frac{w_{m}(x)}{w\left(X_{c}\right)}\right)\right)^{2} \mathrm{~d} x=0.273 \mathrm{~N} / \mathrm{m} .
$$

Therefore, the equivalent mass and the equivalent rigidity of the beam according to expressions of (3.20) and (3.27) are:

$$
\begin{aligned}
& m_{c}=0.313 \mathrm{~kg}, \\
& c_{c}=2.747 \mathrm{~N} / \mathrm{m} .
\end{aligned}
$$

\section{The results of the experimental study}

The reliability of the calculated values of the $m_{c}$ equivalent mass and the $c_{c}$ equivalent rigidity can be determined by upholding of the value of the partial frequency of the $\omega_{p}$ continual segment. Due to the 
established technical requirements, the partial frequency should be of $\omega_{p}=95.672 \mathrm{rad} / \mathrm{s}$. According to calculations using Eq.(3.29) and Eq.(3.28), the partial frequency is:

$$
\omega_{p}=\sqrt{\frac{c_{c}}{m_{c}}}=93.707 \mathrm{rad} / \mathrm{s},
$$

the result coincides quite precisely with the set parameter.

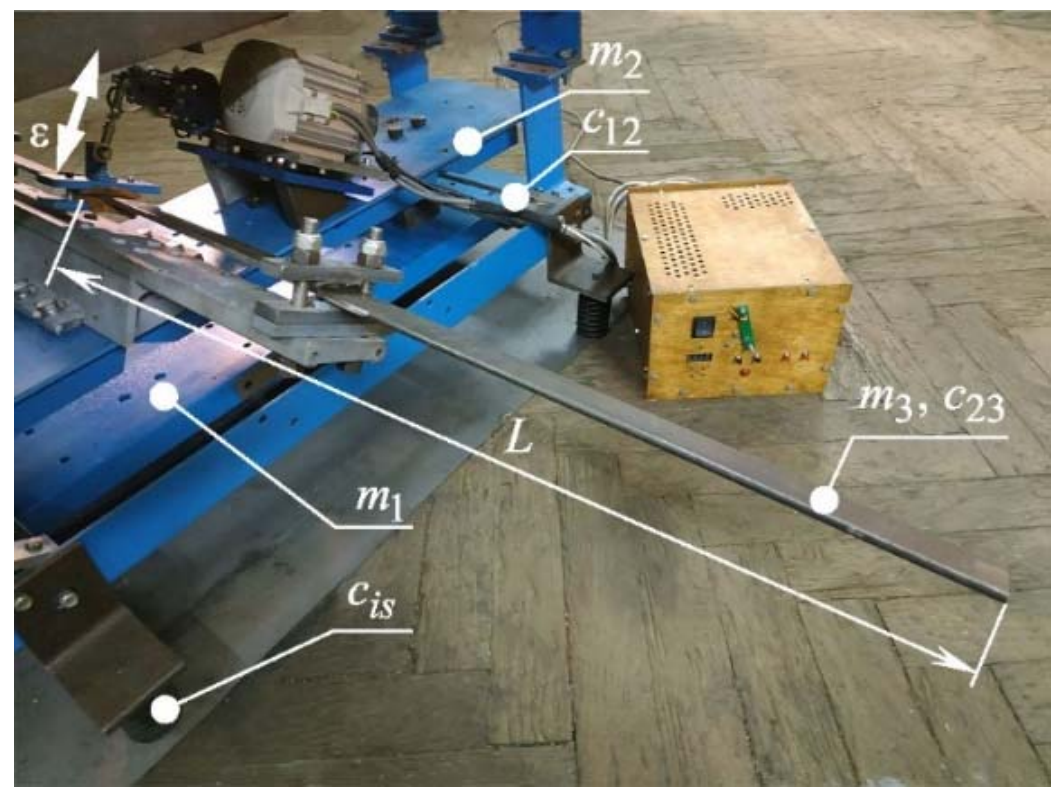

(a)

Vibration cyclequantity

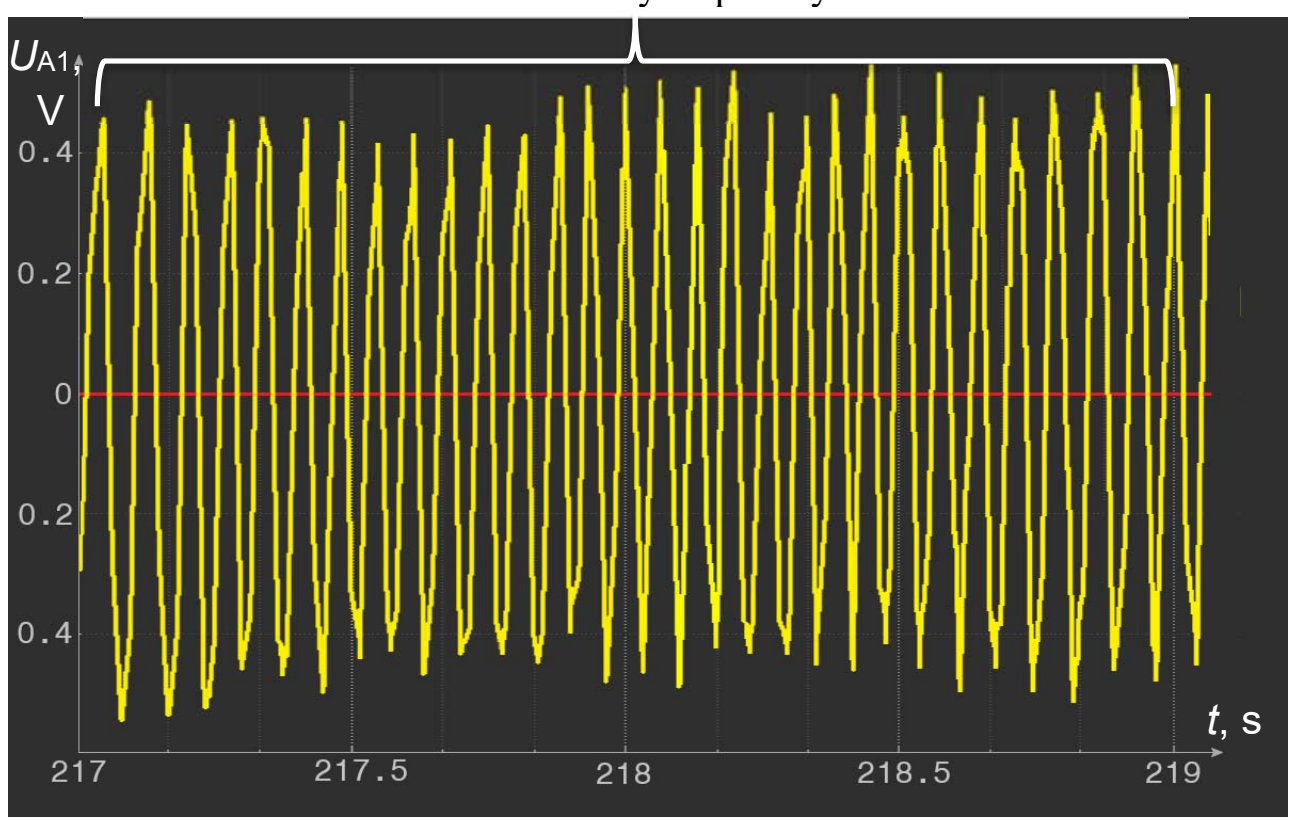

(b)

Fig.4. Experimental sample of the (a) conveyor-separator and amplitude-time characteristic of the (b) intermediate mass (the amplitude value of vibrations is read from the sensor in volts). 
The continual segment in the form of the beam with the geometric parameters given in the article is implemented in an inter-resonance vibrating machine. The nature of vibrations of the intermediate mass, which was set in motion from the continual segment with vibration at its own (partial) frequency, was experimentally indicated. An experimental sample of the continual segment of the inter-resonance vibrating machine is shown in Fig.4a. Inertial parameters of sensors connected to the $m_{2}$ mass practically do not affect the vibrating system because of $m_{2}=62.1 \mathrm{~kg}$.

The time characteristics of the $m_{2}$ mass movement during vibrations of the beam at its own frequency are shown in Fig.4b. The value of the first natural cyclic frequency of vibrations of the beam was determined from the ratio of the number of peaks to the time interval, which is defined as the difference of 219-217, Fig.4b:

$$
v_{p e}=\frac{\text { vibration cycle quantity }}{\text { time interval }} \approx \frac{29.4}{219-217} \approx 14.7 \mathrm{~Hz} .
$$

The circular frequency of the beam, according to Eq.(4.2), will be $\omega_{p e} \approx 92.36 \mathrm{rad} / \mathrm{s}$. The obtained value is consistent with the results of simulation $\left(\omega_{p}=93.707 \mathrm{rad} / \mathrm{s}\right)$.

To analyze quantitatively of the consistency between the simulation results and the experimental data, the frequency error is calculated:

$$
\varepsilon_{p}=\left|\frac{\omega_{p e}-\omega_{p}}{\omega_{p e}}\right| \cdot 100 \%=\left|\frac{92.36-93.707}{92.36}\right| \cdot 100=1.46 \% .
$$

The frequency error does not exceed $2 \%$, which confirms the reliability of theoretical studies.

\section{Conclusions}

For inter-resonance vibration machines, the developed analytical model renders it possible to synthesize continual segments, the properties of which are consistent with the relevant sections of discrete systems. Using the developed model, the two defining parameters of the oscillating system of the vibrating machine are studied, namely: the inertial parameter of reactive mass and appropriate rigidity of the elastic coupling. These operation factors are revealed only in dynamic processes and are clearly not included in the parameters of the continual segment. In order to take into account these parameters the reference point of the continual segment passing through its centre of velocity is studied. The inertial parameter of the reactive mass and the rigidity of the elastic coupling were studied by the Rayleigh-Ritz method. The reliability of the results of theoretical research was confirmed experimentally and the parameters of the partial frequency of the continual segment were determined.

\section{Nomenclature}

$$
\begin{aligned}
A, B, C, D & - \text { arbitrary constants, }[-] \\
b_{r} & - \text { beam thickness, }[\mathrm{m}] \\
c_{c} & - \text { equivalent rigidity of the beam, }[\mathrm{N} / \mathrm{m}] \\
c_{c l i}, c_{c 2 i}, c_{c 3 i} & - \text { fractions of equivalent rigidities of the beam shearing on left, middle and right sections, respectively, }[\mathrm{N} / \mathrm{m}] \\
c_{c 1}, c_{c 2}, c_{c 3} & - \text { fractions of equivalent rigidities of the beam bending on left, middle and right sections, respectively, }[\mathrm{N} / \mathrm{m}] \\
c_{i s} & - \text { rigidity of vibration isolators, }[\mathrm{N} / \mathrm{m}] \\
c_{12}, c_{23} & - \text { rigidity of elastic couplings that connect the active mass to the intermediate and intermediate mass to the } \\
& \text { reactive mass, respectively, }[\mathrm{N} / \mathrm{m}]
\end{aligned}
$$




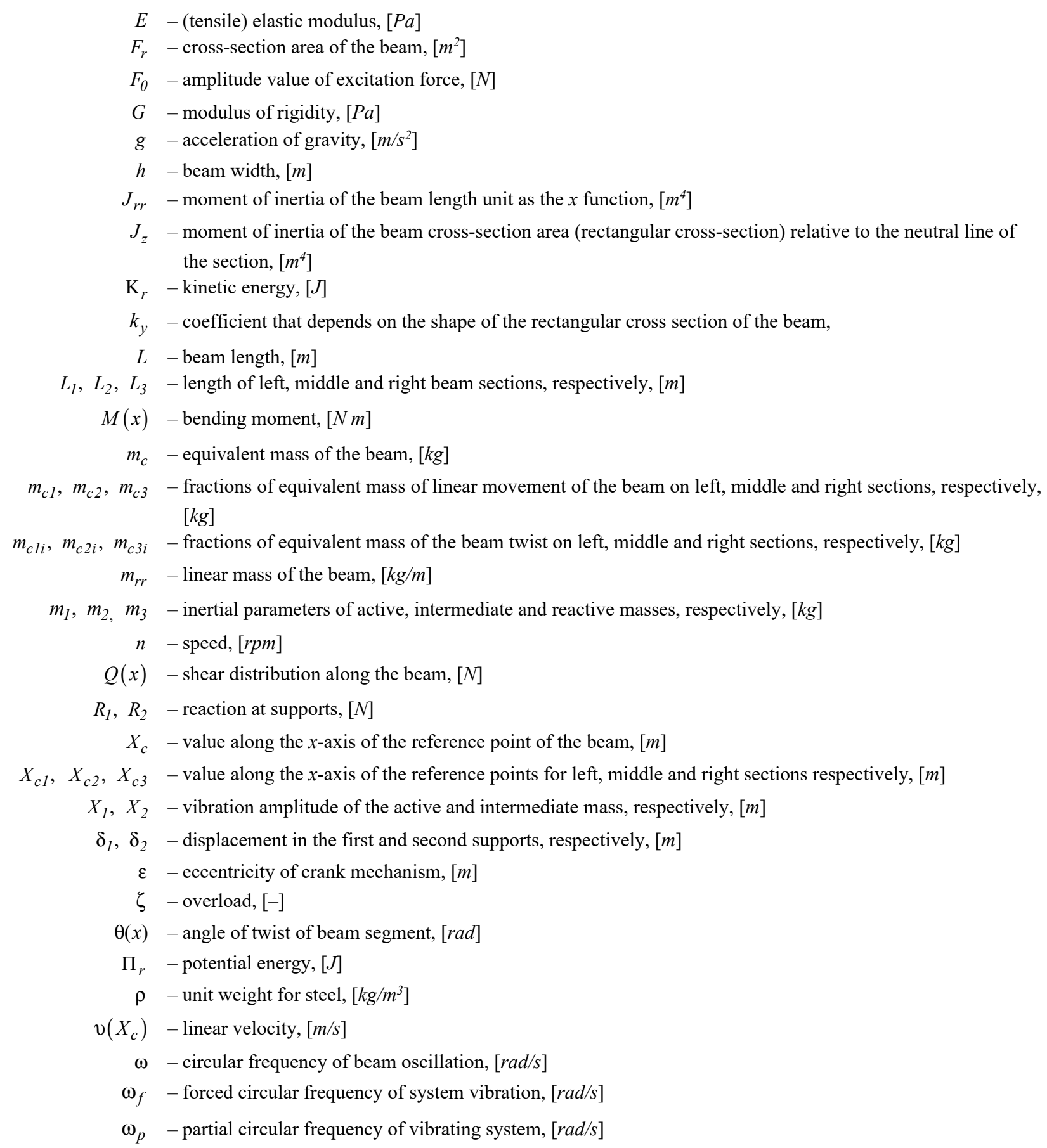

\section{References}

[1] Lanets O.S., Derevenko I.A., Borovets V.M., Kovtonyuk M.M., Komada P., Mussabekov K. and Yeraliyeva B. (2019): Substantiation of consolidated inertial parameters of vibrating bunker feeder.- Przegląd Elektrotechniczny, vol.95, No.4, pp.47-52.

[2] Gursky V.M., Kuzio I.V., Lanets O.S., Kisała P., Tolegenova A. and Syzdykpayeva A. (2019): Implementation of dual-frequency resonant vibratory machines with pulsed electromagnetic drive.- Przegląd Elektrotechniczny, vol.95, No.4, pp.41-46. 
[3] Sharma A.K., Sharma P., Chauhan P.S. and Bhadoria S.S. (2018): Study on harmonic analysis of functionally graded plates using fem.- International Journal of Applied Mechanics and Engineering, vol.23, No.4, pp.941-961.

[4] Jaworski J. W. and Dowell E. H. (2008): Free vibration of a cantilevered beam with multiple steps: comparison of several theoretical methods with experiment.- Journal of Sound and Vibration, vol.312, No.4-5, pp.713-725.

[5] Raju L.S., Raju K. and Rao G.V. (1976): Large amplitude free vibrations of tapered beams.- AIAA Journal, vol.14, No.2, pp.280-282.

[6] Gharaibeh M.A., Obeidat A.M. and Obaidat M.H. (2018): numerical investigation of the free vibration of partially clamped rectangular plates.- International Journal of Applied Mechanics and Engineering, vol.23, No.2, pp.385-400.

[7] Srinivasa C.V., Suresh Y.J. and Prema Kumar W.P. (2014): Experimental and finite element studies on free vibration of skew plates.- International Journal of Applied Mechanics and Engineering, vol.19, No.2, pp.365-377.

[8] Jaroszewicz J. (2017): Natural frequencies of axisymmetric vibrations of thin hyperbolic circular plates with clamped edges.- International Journal of Applied Mechanics and Engineering, vol.22, No.2, pp.451-457.

[9] Zur K.K. (2015): Green's function in frequency analysis of circular thin plates of variable thickness.- Journal of Theoretical and Applied Mechanics, vol.53, No.4, pp.873-884.

[10] Amabili M. and Carra S. (2012): Experiments and simulations for large-amplitude vibrations of rectangular plates carrying concentrated masses.- Journal of Sound and Vibration, vol.331, No.1, pp.155-166.

[11] Buchacz A. (2009): Calculation of flexibility of vibrating beam as the subsystem of mechatronic system by means the exact and approximate methods.- Proceedings in Applied Mathematics and Mechanics, vol.9, No.1, pp.373-374.

[12] Buchacz A. (2011): The supply of formal notions to synthesis of the vibrating discrete-continuous mechatronic systems.- Journal of Achievements in Materials and Manufacturing Engineering, International OCOSCO World Press, vol. 44, No.2, pp.168-178.

[13] Clementi F., Demeio L., Mazzilli C.E.N. and Lenci S. (2015): Nonlinear vibrations of non-uniform beams by the MTS asymptotic expansion method.- Continuum Mech. Thermodyn, vol.27, pp.703-717.

[14] Firouz-Abadi R.D., Haddadpour H. and Novinzadeh A.B. (2007): An asymptotic solution to transverse free vibrations of variable-section beams.- Journal of Sound and Vibration, vol.304, pp.530-540.

[15] Ece M.C., Aydogdu M. and Taskin V. (2007): Vibration of a variable cross-section beam.- Mechanics Research Communications, vol.34, No.1, pp.78-84.

[16] Mahmoud B., Iman P. and Mahdi B. (2011): Analytical study on the vibration frequencies of tapered beams.Latin Ameriacan Journal of Solids and Structures, vol.8. pp.149-162.

[17] Kisa M. and Gurel M. A. (2007): Free vibration analysis of uniform and stepped cracked beams with circular cross sections.- International Journal of Engineering Science, vol.45, No.2-8, pp.364-380.

[18] Lee B.K., Lee J.K., Lee T.E. and Kim S.G. (2002): Free vibrations of tapered Beams with general boundary condition.- (KSCE) Journal of Civil Engineering, vol.6, No.3, pp.283-288.

[19] Shin Y. J., Kwon K. M. and Yun J. H. (2008): Vibration analysis of a circular arch with variable cross section using differential transformation and generalized differential quadrature.- Journal of Sound and Vibration, vol.309, No.1-2, pp.9-19.

[20] Lee J.W. (2016): Free vibration analysis using the transfer-matrix method on a tapered beam.- Journal of Computers and Structures, vol.164, pp.75-82.

[21] Vescovini R., Dozio L., D’Ottavio M. and Polit O. (2018): On the application of the Ritz method to free vibration and buckling analysis of highly anisotropic plates.- Composite Structures, vol.192, pp.460-474.

[22] Dozio L. (2011): On the use of the trigonometric Ritz method for general vibration analysis of rectangular Kirchhoff plates.- Thin-Walled Structures, vol.49, No.1, pp.129-144.

[23] Yuan J. and Dickinson S. M. (1992): The flexural vibration of rectangular plate systems approached by using artificial springs in the Rayleigh-Ritz method.- Journal of Sound and Vibration, vol.159, No.1, pp.39-55. 
[24] Kumar Y. (2018): The Rayleigh-Ritz method for linear dynamic, static and buckling behavior of beams, shells and plates: A literature review.- Journal of Vibration and Control, vol.24, No.7, pp.1205-1227.

[25] Rahbar-Ranji A. and Shahbaztabar A. (2016): Free vibration analysis of moderately thick rectangular plates on Pasternak foundation with point supports and elastically restrained edges by using the Rayleigh-Ritz method.- Journal of Failure Analysis and Prevention, vol.16, pp.1006-1023.

[26] Mazanoglu K. (2017): Natural frequency analyses of segmented Timoshenko-Euler beams using the RayleighRitz method.- Journal of Vibration and Control, vol.23, No.13, pp.2135-2154.

[27] Babakov I. M. (1968): Theory of Vibration.- Leningrad, Nauka.

Received: October 30, 2020

Revised: December 17, 2020 